\title{
Potential value of intraoperative Doppler colour flow mapping in operations for complex intracardiac shunting
}

\author{
G R SUTHERLAND, S BALAJI, J L MONRO \\ From the Department of Paediatric Cardiology, Wessex Cardiothoracic Unit, Southampton Genera! Hospital, \\ Southampton
}

SUMMARY An eight year old boy presented with multiple residual intracardiac shunts and considerable persisting haemolytic anaemia five years after the repair of a single perimembranous ventricular septal defect. Preoperative transthoracic colour flow mapping showed an "acquired" left ventricular right atrial shunt and three small residual ventricular septal defects around the patch. These had been poorly visualised by both angiography and conventional cross sectional echocardiography and pulsed and continuous wave Doppler. Clearly, to stop the haemolysis effectively, closure of all residual intracardiac shunts was required. Intraoperative pre-bypass colour flow mapping was successfully used to confirm the precise number and nature of the defects and more importantly studies after bypass were used to confirm immediate closure of all defects.

There can be major diagnostic problems when multiple complex intracardiac shunts are present. The information provided by angiography is often inadequate. Cross sectional echocardiography with integrated colour flow mapping can reliably define the origin and extent of such complex disturbances. ${ }^{1-3}$ We report a case that shows the unique diagnostic advantages of the technique, but more importantly we describe its intraoperative use in confirming the closure of multiple defects, one or more of which were causing severe haemolysis.

\section{Case report}

An eight year old boy was referred five years after attempted surgical repair of a single perimembranous ventricular septal defect with a concomitant aortic valvotomy. Considerable haemolytic anaemia had been noted during the fifth postoperative month.

Requests for reprints to Dr G R Sutherland, Department of Cardiac Ultrasound, Thoraxcenter, Ba 300, Erasmus University, 3000 DR Rotterdam, The Netherlands.
Clinical examination suggested a small residual ventricular septal defect. Treatment with iron and folic acid supplements was started. Over the next four years his haemoglobin concentration remained normal but a reticulocytosis of $20 \%$ persisted.

On admission, examination showed signs of a residual ventricular septal defect and mild aortic stenosis. Duplex scanning (imaging + pulsed and continuous wave Doppler) suggested only a single ventricular septal defect at the subaortic end of the patch (transseptal peak jet velocity of $5 \mathrm{~m} / \mathrm{s}$ ). Colour flow mapping showed numerous residual discrete jets-one from the left ventricle to right atrium ("acquired" Gerbode type defect) and three peripatch transseptal jets from the left to right ventricle. Subsequent left ventricular angiography from appropriately angled views suggested only two ventricular septal defects but failed to show the "acquired" Gerbode defect.

Successful surgical closure of all defects was clearly essential to stop the haemolytic anaemia. We decided to carry out "intraoperative angiography" using colour flow mapping to confirm the sites of the defects and to confirm defect closure. Intraoperative 


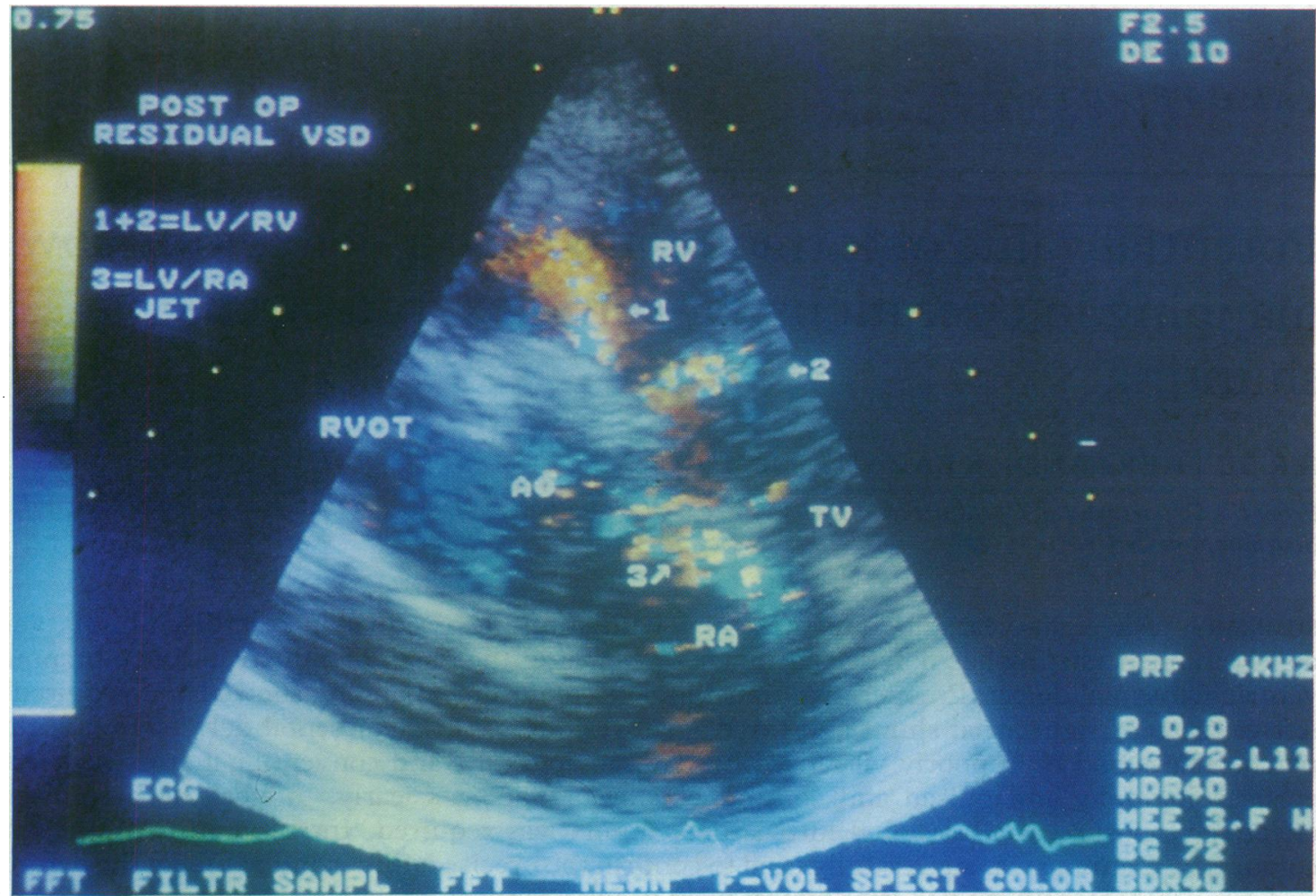

Fig 1 Intraoperative pre-repair colour flow map in the short axis, right ventricular view showing three residual intracardiac shunts. Numbers 1 and 2 are interventricular shunts and 3 is a left ventricle to right atrial shunt (a further more apical ventricular septal defect was present at the lower end of the patch that could not be included in this view). Blood flow towards the transducer is red and flow away from the transducer is blue. Turbulence (that is a high velocity flow disturbance) is encoded as a mosaic of yellow. $R A$, right atrium; $R V$, right ventricle; $R V O T$, right ventricular outflow tract; Ao, aorta; $L V$, left ventricle.

colour flow mapping was performed with a Toshiba SSH-65A. The transducers $(5 \mathrm{MHz}$ for imaging, and $2.5 \mathrm{MHz}$ for colour flow mapping) were placed into long ( 2 metre) sterile plastic bags containing ultrasound gel which were then placed directly on the beating heart. Intraoperative colour flow mapping confirmed the presence of the "acquired" Gerbode defect and showed three further restrictive ventricular septal defects all around the margins of the patch (fig 1). All defects were then closed with Teflon pledgets. Epicardial colour flow mapping was repeated with the patient off cardiopulmonary bypass. This showed normal low velocity laminar flow within the right heart with no residual turbulence in either right atrium or right ventricle (fig 2). A transthoracic colour flow mapping study on the fifth postoperative day confirmed the continuing integrity of the repair.

Six months after operation further colour flow mapping studies showed no residual septal defect.
Since operation both the haemoglobin concentration and reticulocyte count have remained normal.

\section{Discussion}

It was important in this patient to identify all the residual defects before operation and then to confirm successful surgical closure intraoperatively because any or all of the defects could have contributed to the haemolysis. In this patient intraoperative epicardial colour flow mapping proved to be more sensitive than either duplex scanning or angiography in the preoperative assessment. Intraoperative colour flow mapping proved to be easy to perform, sensitive, and provided a rapid guide to the surgeon as to the site of the residual defects and the subsequent adequacy of his repair. This case shows the great value of this technique in the management of patients with multiple complex intracardiac shunts. 


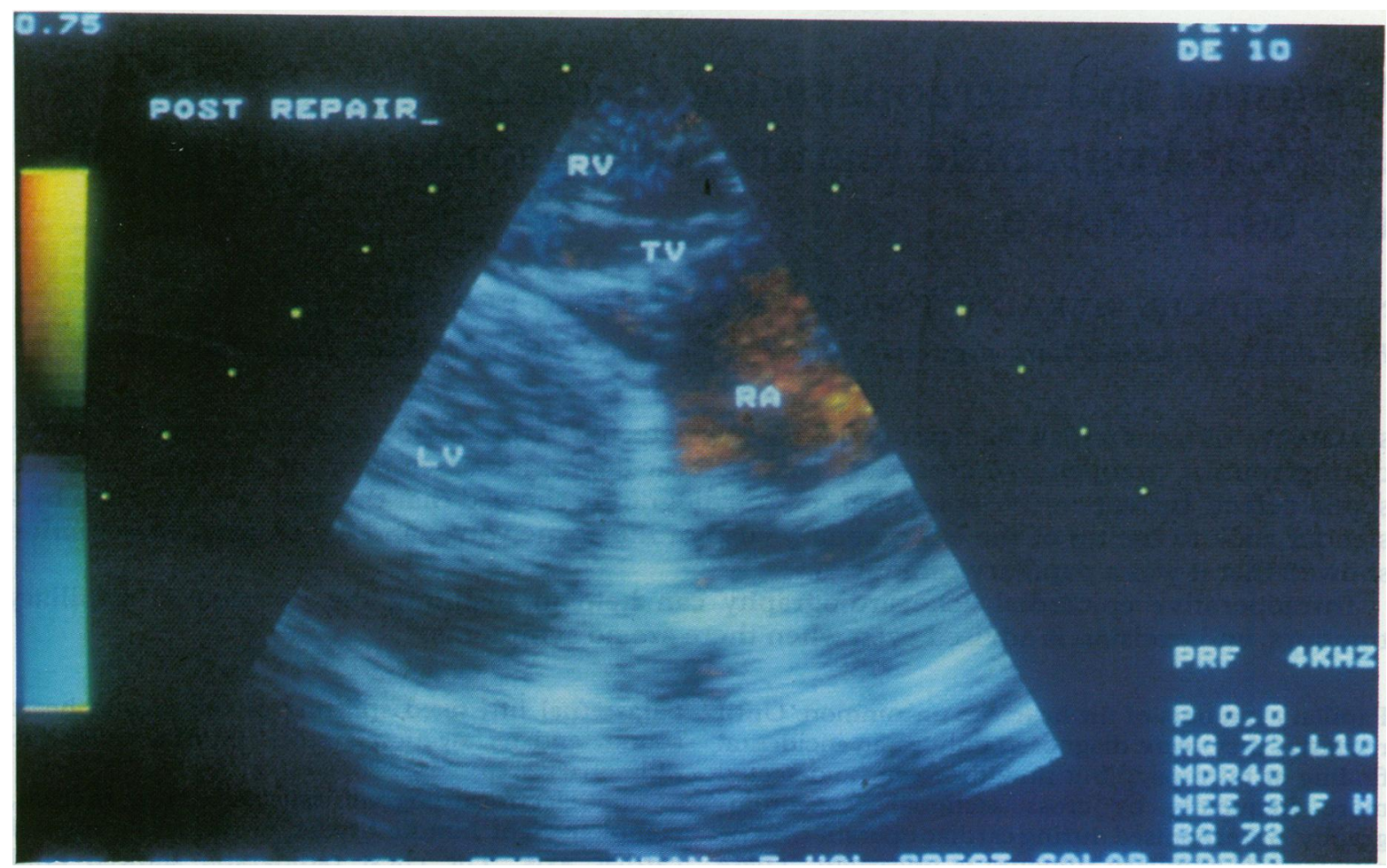

Fig 2 Foreshortened four chamber view of intraoperative post-repair colour flow map showing no residual turbulent flow within either the right ventricle or right atrium. No residual intracardiac shunts. The left ventricle cavity is small because of hypovolaemia immediately after the patient came off cardiopulmonary bypass. See legend to fig 1 for abbreviations.

\section{References}

1 Takamoto S, Kyo S, Adachi $\mathrm{H}$, et al. Intraoperative colour flow mapping by real-time two-dimensional Doppler echocardiography for evaluation of valvular and congenital heart disease and vascular disease. $J$ Thorac Cardiovasc Surg 1985;90:802-12.

2 Ortiz E, Robinson PJ, Deanfield JE, Franklin R,
Macartney FJ, Wyse RKH. Localisation of ventricular septal defects by simultaneous display of superimposed colour Doppler and cross sectional echocardiographic images. Br Heart J 1985;54:53-60.

3 Ludomirsky A, Huhta JC, Vick GW, Murphy DJ, Danford DA, Morrow WR. Colour Doppler detection of multiple ventricular septal defects. Circulation 1986;74:1317-22. 